\title{
Optimization of Resource Allocation for the Downlink of Multiuser MISO-OFDM Systems
}

\author{
${ }^{\dagger}$ Lidong Zhu and ${ }^{\dagger}$ Kwan L. Yeung \\ "Department of Electrical and Electronic Engineering, The University of Hong Kong, Hong Kong, PRC. \\ ${ }^{*}$ National Key Laboratory of Communications, University of Electronic Science and Technology of China, Chengdu, China \\ Tel: (852) 2857-8493 Fax: (852) 2559-8738 \\ Email: \{zld@uestc.edu.cn, kyeung@eee.hku.hk\}
}

\begin{abstract}
In this paper, we investigate the optimization problem of resource allocation in downlink of multiuser MISO-OFDM system. Multiple users with different BER and minimum transmission rate requirements are considered. We propose a novel heuristic allocation algorithm (HAA) of radio resource, which minimizes the total transmit power of the base station while meeting individual users' $Q \mathrm{OS}$ requirements. The proposed algorithm combines antenna selection, subcarrier, bit and power allocation together, pre-estimating number of subcarriers assigned to each user and number of bits loaded for each subcarrier to reduce search number, reducing about $8 \mathrm{~dB}$ average bit SNR comparing with fixed allocation algorithm (FAA), and acquiring asymptotic average bit SNR of optimal allocation algorithm (OAA) with much lower complexity.
\end{abstract}

\section{INTRODUCTION}

Multiple-input multiple-output (MIMO) transmission with multiple antennas can increase the system capacity by multiplexing gain and improve the reception reliability of receivers by spatial diversity gain. It is a promising technique to meet the growing demand of capacity in the future wideband wireless communication systems. In practice, due to the size and cost limitation, it is difficult to deploy multiple antennas in mobile terminals. Suppose that there are multiple antennas at the base station and one at each mobile terminal, then MIMO becomes MISO [1].

Orthogonal Frequency Division Multiplexing (OFDM) is an effective technique for combating frequency selective fading caused by multi-path fading channel. An OFDM system can assign different modulation schemes for each subcarrier according to its signal to noise ratio (SNR). Adaptive bit-loading can be used to allocate more bits for subcarriers with higher SNR. The combining use of MIMO and OFDM can significantly improve the performance of wireless communication systems. However, since the channel states of multiuser MIMO-OFDM systems are time varying, static resource allocation is not optimal, and how to allocate spatial and spectral resources dynamically according to the time varying channels is one of the most challenging research topics.

There are different optimization techniques for multiuser OFDM systems, which can be classified into two types: margin adaptive and rate adaptive. The objective of margin adaptive is to achieve the minimum overall transmit power while not violating the constraints on user's data rate [2-4]. The objective of rate adaptive is to maximize each user's data rate for a given total transmit power [5].

Existing work on subcarrier, bit and power allocation for multiuser OFDM systems usually assumes perfect channel state information is known by both transmitter and receiver. The associated resource optimization problem is nonlinear and is thus difficult to solve. Notably, the resource optimization for $\mathrm{SISO} / \mathrm{OFDM}$ systems is studied in [2-5], and one solution is to convert the nonlinear optimization problem into an integer linear programming [5]. Then several suboptimal resource allocation algorithms are proposed through linear programming relaxation. Lagrange multipliers are used to solve the optimization problem but the complexity is still high. In [6-8], multiuser MIMO/OFDM systems are considered. With the rule that a subcarrier is to be used by at most one user, antenna selection is combined with subcarrier, bit and power allocation. A general approach is to construct a $Q \times R$ matrix $(Q$ is the number of transmit antennas at the base station and $R$ is the number of receive antenna for each user), and each entry $H_{k, n}$ denotes the channel gain vector for the $n$-th subcarrier assigned to the $k$-th user. By singular decomposition, the largest eigenvalue of the matrix is found, and the transmit power from the base station to user $k$ on subcarrier $n$ is determined. In [9-10], subcarrier sharing by multiple users is allowed. This is achieved by precoding at the transmitter of the base station using dirty paper coding or zero-forcing beamforming, cochannel interference from multiple users can be mitigated effectively.

In this paper, we focus on the optimization of resource allocation in the downlink of a multiuser MISO-OFDM system under the assumption that a subcarrier can only be used by a single user. The users are divided into different classes of services by their requirements of minimum transmission rate and bit error rate (BER). An efficient heuristic algorithm for joint optimization of antenna selection, subcarrier allocation, bit-loading and power allocation is proposed. We follow the margin adaptive approach [2-4] of minimizing the total transmit power of the base station while meeting the QoS requirements of individual users. [2-4] considers the constraint of user data rate but doesn't consider different BER requirement of individual user, and assume BER is the same for all users in the simulation. Our algorithm considers the constraints of BER and minimum transmission rate of individual users simultaneously. The basic idea of our proposed algorithm is to pre-estimate the number of subcarriers to be assigned to each user according to the ratio of minimum transmission rate of each user to the sum of minimum transmission rate of all users, and the number of bits to be assigned to each subcarrier according to the ratio of the channel gain of a subcarrier to the average channel gain of all users. By doing so, we can significantly reduce the searching effort for finding a good solution, while still attaining close to optimal performance. 


\section{PROBLEM FORMULATION}

Consider a multiple-input and single-output (MISO) system with one base station (BS). There are $Q$ transmit antennas at the base station and a single receive antenna at each user terminal. The BS has $N$ subcarriers and serves $K$ users. The users are distributed uniformly in the coverage area of the base station. Let $N>K$. The system model is shown in Fig. 1. At the transmitter of the BS, the data from $K$ users are fed into the module for antenna selection, subcarrier, bit, and power allocation, which allocates bits to subcarriers assigned to different users. Note that an OFDM symbol is composed of multiple subcarriers, and the transmitter sends an OFDM symbol at each time slot. We assume that each user has the capability to estimate the channel quality and report the channel state information to the BS in every time slot. That is to say, both transmitter and receiver have perfect channel state information (CSI), and the channel state changes little during one transmission. According to the channel gain of each subcarrier and the QoS requirement of each user, specific modulation scheme is selected. The inverse fast Fourier transform (IFFT) transforms the symbols into time-domain samples. These samples are transmitted through antennas over fading channels when the parallel-to-serial conversion completes and the cyclic prefix is inserted for mitigating the inter-symbol interference.
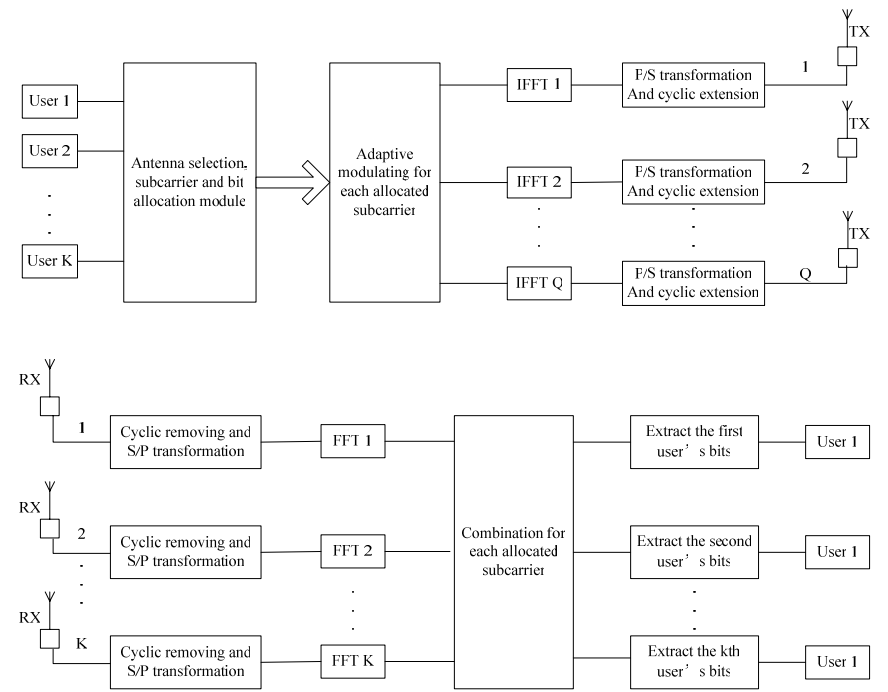

Fig. 1. The diagram of the system model

Let $b_{k, n}$ denote the transmission bits per symbol and $p_{k, n}$ the transmit power for subcarrier $n$ assigned to user $k$, then $p_{k, n}$ is given by

$$
p_{k, n}=f_{k}\left(\mathrm{~b}_{\mathrm{k}, \mathrm{n}}\right) /\left|\mathrm{h}_{\mathrm{k}, \mathrm{n}}\right|^{2}
$$

where $f_{k}\left(b_{k, n}\right)$ is the receive power (in energy per symbol) in a subcarrier for reliable reception of $b_{k, n}$ bits when the channel gain is equal to unity. $\left|h_{k, n}\right|^{2}$ is the channel gain of subcarrier $n$ assigned to user $k$.

For MISO-OFDM systems, $\left|h_{k, n}\right|^{2}$ is a $1 \times Q$ vector, or

$$
\left(\left|h_{k, n}^{(1)}\right|^{2},\left|h_{k, n}^{(2)}\right|^{2}, \cdots,\left|h_{k, n}^{(q)}\right|^{2},\left|h_{k, n}^{(q+1)}\right|^{2} \cdots,\left|h_{k, n}^{(Q)}\right|^{2}\right),
$$

where $h_{k, n}^{(q)}$ is the channel frequency response of subcarrier $n$ for user $k$ from the $q$-th antenna at the BS, and $\left|h_{k, n}^{(q)}\right|^{2}$ is the corresponding channel gain.

From [11], the receive power $f_{k}\left(b_{k, n}\right)$ in (1) can be rewritten as

$f_{k}\left(b_{k, n}\right)=\left(N_{0} / 3\right) \cdot\left[Q^{-1}\left(B E R_{k}^{*} / 4\right)\right]^{2} \cdot\left(2^{b_{k, n}}-1\right)$

where

$$
Q(x)=\frac{1}{\sqrt{2 \pi}} \int_{x}^{\infty} e^{-t^{2} / 2} d t
$$

In (2), $N_{0}$ is the single-sided power spectrum density and $B E R_{k}^{*}$ is the required bit error rate for user $k$.

Combining (1) and (2), we have

$$
p_{k, n}=\left(N_{0} / 3\right)\left[Q^{-1}\left(B E R_{k}^{*} / 4\right)\right]\left(2^{b_{k, n}-1}\right) /\left|h_{k, n}\right|^{2}
$$

Then the total transmit power at the base station is given by

$$
P_{\text {total }}=\sum_{n=1}^{N} \sum_{k=1}^{K} p_{k, n}=\sum_{\mathrm{n}=1}^{\mathrm{N}} \sum_{\mathrm{k}=1}^{\mathrm{K}} f_{k}\left(b_{k, n}\right) /\left|h_{k, n}\right|^{2}
$$

Let $R_{k}$ and $B E R_{k}$ be the allocated transmission rate and bit error rate of user $k$, and $B E R_{k}^{*}$ and $R_{k}^{*}$ are user $k$ 's requirements. We use $\beta_{k, n}=1$ to indicate subcarrier $n$ is assigned to user $k$, and $\beta_{k, n}=0$ otherwise. The data rate allocated to user $k$ is

$$
R_{k}=\sum_{n=1}^{N} \beta_{k, n} b_{k, n}
$$

To minimize the total BS transmit power, the optimization problem can be formulated as

$$
\min P_{\text {total }}=\left.\min \sum_{k=1}^{K} \sum_{n=1}^{N}\left[\frac{1}{3} N_{0}\left[Q^{-1}\left(B E R_{k}^{*} / 4\right)\right]^{2}\left(2^{b_{k, n}}-1\right)\right]|| h_{k, n}\right|^{2}
$$

Subject to the following constraints:

$$
\left\{\begin{array}{l}
B E R_{k} \leq B E R_{k}^{*}, \forall \mathrm{k} \in(1,2, \cdots, \mathrm{K}) \\
R_{k} \geq R_{k}^{*}, \forall \mathrm{k} \in(1,2, \cdots, \mathrm{K}) \\
p_{k} \geq 0, \mathrm{k} \in(1,2, \cdots, \mathrm{K})
\end{array}\right.
$$

We use the concept of Average Bit SNR (short for ABSNR), which is defined as the ratio of the average transmit power (in energy per bit) to the noise power spectrum density [4], or

$$
\operatorname{ABSNR}=\left(P_{\text {total }} / \sum_{k=1}^{K} R_{k}\right) /(\Omega / W)
$$

where $\Omega$ is noise power, $W$ is the system bandwidth and $\Omega / W$ is noise power spectrum density. 
Substituting (5) and (6) into (9), we have

$$
A B S N R=\left[\left(\sum_{n=1}^{N} \sum_{k=1}^{K} f_{k}\left(b_{k, n}\right)\left|h_{k, n}\right|^{2}\right) /\left(\sum_{k=1}^{K} \sum_{n=1}^{N} \beta_{k, n} b_{k, n}\right)\right] /(\Omega / W)
$$

Insert (2) into (10), then

$$
\operatorname{ABSNR}=\frac{\sum_{n=1}^{N} \sum_{k=1}^{K}\left[\left[\left(N_{0} / 3\right)\left[Q^{-1}\left(B E R_{k}^{*} / 4\right)\right]^{2}\left(2^{b_{k, n}}-1\right)\right] /\left|h_{k, n}^{2}\right|^{2}\right]}{(\Omega / W) \cdot\left[\sum_{k=1}^{K} \sum_{n=1}^{N} \beta_{k, n} b_{k, n}\right]}
$$

Given $\Omega / W$ and minimum transmission rate requirements of each user, $A B S N R$ is proportional to $P_{\text {total }}$. So minimizing $P_{\text {total }}$ in (7) is equivalent to the minimization of ABSNR.

$$
\min \mathrm{ABSNR}=\min \frac{\sum_{n=1}^{N} \sum_{k=1}^{K}\left[\left[\frac{N_{0}}{3} \cdot\left[Q^{-1}\left(B E R_{k}^{*} / 4\right)\right]^{2}\left(2^{b_{k, n}}-1\right)\right] /\left|h_{k, n}^{2}\right|^{2}\right]}{(\Omega / W) \cdot\left[\sum_{k=1}^{K} \sum_{n=1}^{N} \beta_{k, n} b_{k, n}\right]}
$$

Subject to the constraints given by equation (8).

From equation (12) we know that ABSNR is related with $B E R_{k}^{*}$ and $b_{k, n}$. For convenience of analysis, we suppose $N_{0}=1, \Omega / W=1$ in the following.

\section{RESOURCE ALLOCATION ALGORITHM}

In a multiuser MISO-OFDM system, the optimization problem in (12) involves antenna selection, subcarrier allocation and bit loading for each subcarrier. It can be solved by exhaustive search or suboptimal allocation algorithms as in [2-4]. But the computational complexity involved is formidable. In this section, we propose an efficient heuristic algorithm (HAA) for suboptimal performance. Our heuristic algorithm consists of five steps:

- pre-estimate firstly the number of subcarriers to be assigned to each user;

- select antenna and subcarriers for each user based on the estimated number of subcarriers;

- pre-estimate the number of bits assigned to each subcarrier and adjust the number of bits according to the requirement of the user's minimum transmission rate and minimum transmit power, then load bits for each subcarrier;

- assign residual subcarriers to the user whose QoS requirement is not satisfied;

- assign power to each subcarrier according to (4).

We elaborate on each step in more details below.

\section{A. Estimating number of subcarriers}

Our method of estimating number of subcarriers is different from [2-4]. [2] determines number of subcarriers by $n_{k}=\operatorname{round}(N / K)$. [3] assigns the initial number of subcarriers to user $k$ according to $n_{k}^{\text {initial }}=\left\lceil R_{k} / R_{\max }\right\rceil, R_{\max }$ is the maximum number of bits a subcarrier can be loaded, corresponding to best channel quality. Both [2] and [3] don't consider the difference of channel gain and minimum data rate requirement for individual users. [4] determines subcarriers for each user by Lagrange multipliers but with high complexity. Considering the difference of minimum transmission rate for each user, we estimate number of subcarriers as below.

Let $n_{k}^{\prime}$ be the number of subcarriers pre-assigned to user $k$, and it is calculated according to the minimum transmission rate requirement of all the users.

$$
n_{k}^{\prime}=\left\lfloor\left(N \cdot R_{k}^{*}\right) / \sum_{k=1}^{K} R_{k}^{*}\right\rfloor
$$

Note that $R_{k}^{*}$ is the required minimum transmission rate of user $k$.

\section{B. Allocating subcarriers and antennas to each user}

When allocating subcarriers and antennas, we should follow the rule that a subcarrier is shared by only one user, and the subcarrier is allocated to the user with the highest channel gain. Besides, a subcarrier can only be assigned to a user once, and the subcarrier can only be transmitted by one antenna each time. The detailed process is described below.

\section{i . Initialization}

There are $\mathrm{Q}$ antennas. For random antenna $\mathrm{q}, q \in\{1,2, \cdots, Q\}$, we first construct a $K \times N$ channel gain matrix $G_{K \times N}^{(q)}$, where

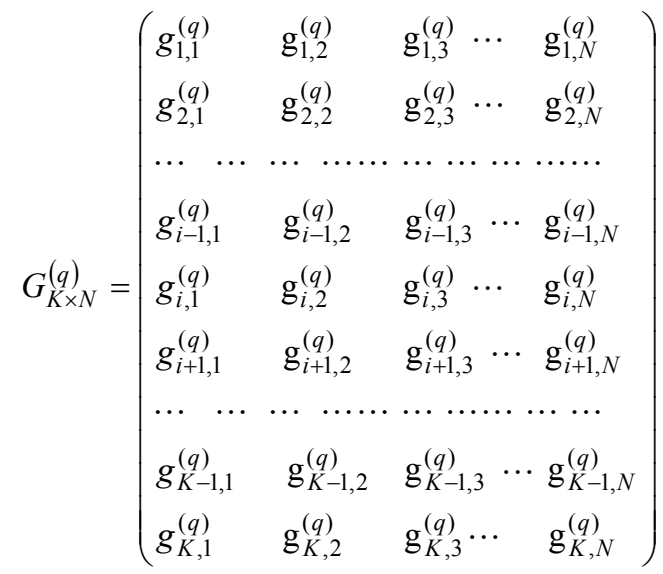

Each entry $g_{k, n}^{(q)}$ denotes the channel gain of subcarrier $n$ for user $k$ at the $q$-th antenna. Specifically, we define $g_{k, n}^{(q)}=\left|h_{k, n}^{(q)}\right|^{2} / \sigma_{k, n}^{2}$, where $\sigma_{k, n}^{2}$ is the variance of the additive white Gaussian noise in subcarrier $n$ for user $k$. As $N_{0}=1$, let $\sigma_{k, n}^{2}=N_{0}$, so $g_{k, n}^{(q)}=\left|h_{k, n}^{(q)}\right|^{2}$.

Suppose the number of subcarriers assigned to user $k$ is $n_{k}$, and the sets of subcarriers assigned to user $k$ is $s_{k}$; let the 
initial value of $n_{k}$ be zero, and the initial value of $s_{k}$ is null set, then

$$
\begin{aligned}
& n_{k}=0, \forall k \in(1,2, \cdots, \mathrm{K}) \\
& s_{k}=\phi, \forall k \in(1,2, \cdots, \mathrm{K})
\end{aligned}
$$

\section{ii. Subcarriers allocation}

There are $K$ elements for each column of matrix $G_{K \times N}^{(q)}$

For random column $n, \quad n \in(1,2, \cdots, \mathrm{N})$, we find the maximum element of the column and record the index of the element.

For subcarrier $n$, the possible channel gains are composed of the elements of the $n$-th column of matrix $G_{K \times N}^{(q)}$. As there are $Q$ antennas corresponding to $Q$ matrixes, so there exist $K Q$ possible channel gains. Subcarrier $n$ is assigned to the user with the highest channel gain at the $n$-th column of the $Q$ matrixes. Given the initial value of number of subcarriers assigned to user $k$ is zero, $n_{k}=0$. For subcarrier $n$, the search process is described below.

$$
\begin{aligned}
& \text { for } q=1: Q \\
& \qquad g_{q}=\max \left\{\mathrm{g}_{1, \mathrm{n}}^{(\mathrm{q})}, \mathrm{g}_{2, \mathrm{n}}^{(\mathrm{q})}, \mathrm{g}_{3, \mathrm{n}}^{(\mathrm{q})}, \cdots, \mathrm{g}_{\mathrm{k}-1, \mathrm{n}}^{(\mathrm{q})}, \mathrm{g}_{\mathrm{k}, \mathrm{n}}^{(\mathrm{q})}, \cdots \mathrm{g}_{\mathrm{K}-1, \mathrm{n}}^{(\mathrm{q})}, \mathrm{g}_{\mathrm{K}, \mathrm{n}}^{(\mathrm{q})}\right\} \text {; } \\
& \text { end } \\
& q^{*}=\arg \max _{\mathrm{q}}\left\{\mathrm{g}_{1}, \mathrm{~g}_{2}, \cdots, \mathrm{g}_{\mathrm{q}-1}, \mathrm{~g}_{\mathrm{q}}, \cdots, \mathrm{g}_{\mathrm{Q}}\right\} ; \\
& k^{*}=\arg \max _{\mathrm{k}}\left\{\mathrm{g}_{1, \mathrm{n}}^{\left(\mathrm{q}^{*}\right)}, \mathrm{g}_{2, \mathrm{n}}^{\left(\mathrm{q}^{*}\right)}, \cdots, \mathrm{g}_{\mathrm{k}-1, \mathrm{n}}^{\left(\mathrm{q}^{*}\right)}, \mathrm{g}_{\mathrm{k}, \mathrm{n}}^{\left(\mathrm{q}^{*}\right)}, \cdots, \mathrm{g}_{\mathrm{K}, \mathrm{n}}^{\left(\mathrm{q}^{*}\right)}\right\} ; \\
& \text { if }\left(\mathrm{n}_{\mathrm{k}}<\mathrm{n}_{\mathrm{k}}^{\prime}\right) \\
& \qquad n_{k}=n_{k}+1, s_{k}=s_{k}+\left\{k^{*}\right\} ; \\
& \text { else } \\
& \text { end }
\end{aligned}
$$

Let $n_{k}=n_{k}+1$ after a new assignment and subcarrier is no more assigned to user $k$ when $n_{k}=n_{k}^{\prime}$.

\section{Loading bits for each subcarrier}

After selecting subcarriers for each user, then we assign number of bits for each subcarrier. Before loading bits, we roughly judge whether the total number of bits assigned to user $k$ is enough to meet its requirement of minimum transmission rate, $R_{k} \geq R_{k}^{*}$, and how many bits should be assigned to each subcarrier according to its channel gain. This is different from greedy algorithm [4], B-tighten algorithm [2], and optimal bit loading algorithm [3]. Greedy algorithm assigns bits to the subcarriers one bit at a time from $b_{k, n}=0$, and in each assignment, the subcarrier that requires the least additional power is selected. The algorithm is optimal but with high complexity. The B-tighten algorithm is simplification of greedy algorithm but still has high complexity. The optimal bit
allocation algorithm adopts the US patent invented by Hughes-Hartogs but is still of complexity.

As number of bits loaded for each subcarrier $n$ is limited to an integer set $\{0, \Delta b, 2 \Delta b, \cdots, M \Delta b\}, \Delta b$ is the increment unit of number of bits, and $b_{\text {median }}$ is the median of all elements of the set.

Firstly, according to equation (15), we calculate the average channel gain of all users, denoted by $G_{\text {average }}$.

$$
G_{\text {average }}=\frac{1}{K \cdot N \cdot Q} \sum_{q=1}^{Q} \sum_{k=1}^{K} \sum_{n=1}^{N} g_{k, n}^{(q)}
$$

For subcarrier $n$ of user $k$ from antenna $q, n \in\left\{1,2, \cdots, n_{k}\right\}$, we calculate the ratio of its channel gain to the average channel gain of all users, denoted by $\alpha_{k}$, where

$$
\alpha_{k}=g_{k, n}^{(q)} / \text { Gaverage }
$$

Then we choose the initial value of $b_{k, n}$ according to equation (17).

$$
b_{k, n}= \begin{cases}\mathrm{b}_{\text {median }}+\Delta b, & \alpha_{\mathrm{k}}>t \\ b_{\text {median }}, & \frac{1}{t} \leq \alpha_{k} \leq t \\ b_{\text {median }}-\Delta b, & \alpha_{\mathrm{k}}<\frac{1}{t}\end{cases}
$$

where $t$ is determined by the difference of the average bit SNR caused by the difference of two adjacent integer bits assigned to subcarrier. Suppose the difference is $x \mathrm{~dB}$, that is

$$
x=A B S N R_{\left(b_{k, n}+\Delta b\right)}-\operatorname{ABSNR}\left(b_{k, n}\right) \text {, then } t=10^{x / 10} \text {. }
$$

The final number of bits assigned to subcarrier $n$ of user $k$ should be adjusted according to the requirement of minimum transmission rate of user $k$, but is limited to increase or reduce $\Delta b$ bits with minimum increment of transmit power. By this bit loading method, we reduce number of search effectively.

\section{Allocating the residual subcarriers}

After allocating bits for the subcarriers assigned to each user, we calculate the data rate of each user $k$, denoted by $R_{k}$, according to (6), and compare $R_{k}$ with $R_{k}^{*}$. If $R_{k} \geq R_{k}^{*}$, then there is no need to assign subcarriers to user $\mathrm{k}$ any more. Otherwise, we assign some of the residual subcarriers to user $k$ until $R_{k} \geq R_{k}^{*}$. The allocation process is the same as step $\mathrm{A}, \mathrm{B}$, and $\mathrm{C}$.

\section{E. Allocating power to each subcarrier}

After determining the subcarriers assigned to each user and finishing bit loading for each subcarrier, we calculate the power assigned to each subcarrier according to (4). The total transmit power of the base station is calculated by (5).

\section{IV . NUMERICAL RESULTS AND ANALYSIS}

\section{A. Two Other Algorithms}

For performance comparison, we introduce two other algorithms of resource allocation, which are fixed allocation algorithm (FAA) and optimal allocation algorithm (OAA) separately.

Fixed allocation algorithm (FAA) assigns subcarriers to all users equally without considering the different channel gain of users, each user of the same class of service is allocated the same number of subcarriers, and each subcarrier is loaded with the same number of bits. The advantage of this algorithm is simple to realize, while its disadvantage is too low utilization efficiency of resource.

Optimal allocation algorithm (OAA) adopts exhaustive search method which always assigns the subcarrier to the user with best channel quality and uses greedy algorithm to load bits 
for each subcarrier. The advantage of this algorithm is high utilization efficiency of resource; its disadvantage is too complex to realize because of its exhaustive search of the best channel. The solution is to use Lagrange multiplier to convert nonlinear problem into multiple linear optimization problem. However, each linear optimization still has high complexity.

\section{B. Specification of Simulation Parameters}

In the simulation, two classes of services with different BER and minimum transmission rate requirements are considered which are service A and service B separately. We suppose that the user number of the two types of services is the same. The simulated channels are four multipath frequency selective Rayleigh fading channels with an exponential delay profile. We use the IMT-2000 outdoor to indoor and pedestrian test environment tapped-delay-line parameters suggested by ITU-R M.1225, and choose the parameters of Channel A. All the simulation parameters are given by Table I.

Table I. Simulation Parameters

\begin{tabular}{|c|c|c|c|}
\hline \multicolumn{2}{|c|}{ Carrier Frequency } & \multicolumn{2}{|c|}{$2.0 \mathrm{GHz}$} \\
\hline \multicolumn{2}{|c|}{ Bandwidth } & \multicolumn{2}{|c|}{$5.0 \mathrm{MHz}$} \\
\hline \multicolumn{2}{|c|}{ Number of bits/symbol } & \multicolumn{2}{|c|}{1024} \\
\hline \multicolumn{2}{|c|}{ Number of Subcarriers } & \multicolumn{2}{|c|}{256} \\
\hline \multicolumn{2}{|c|}{ Number of users } & \multicolumn{2}{|c|}{$4 \sim 64$} \\
\hline \multicolumn{2}{|c|}{$\begin{array}{c}\text { Number of transmit } \\
\text { antenna }\end{array}$} & \multicolumn{2}{|c|}{4} \\
\hline \multirow{2}{*}{\multicolumn{2}{|c|}{ BER Requirements }} & Service A & Service B \\
\hline & & $10^{-3}$ & $10^{-5}$ \\
\hline \multirow{2}{*}{\multicolumn{2}{|c|}{$\begin{array}{c}\text { Minimum Transmission } \\
\text { Rate Requirements }\end{array}$}} & Service A & Service B \\
\hline & & $\begin{array}{c}12 \\
\text { bits/symbol }\end{array}$ & $\begin{array}{c}20 \\
\text { bits/symbol }\end{array}$ \\
\hline Tap & $\begin{array}{c}\text { Relative Delay } \\
\text { (ns) }\end{array}$ & \multicolumn{2}{|c|}{$\begin{array}{c}\text { Average Power } \\
\text { (dB) }\end{array}$} \\
\hline 1 & 0 & \multicolumn{2}{|c|}{0} \\
\hline 2 & 110 & \multicolumn{2}{|c|}{-9.7} \\
\hline 3 & 190 & \multicolumn{2}{|c|}{-19.2} \\
\hline 4 & 410 & \multicolumn{2}{|c|}{-22.8} \\
\hline
\end{tabular}

\section{Numerical Results}

In the following we firstly give an example on how to determine number of bits for a subcarrier. We assume the following modulation schemes, non-modulation, QPSK, 16-QAM, 64-QAM and 256-QAM. The corresponding numbers of transmission bits per subcarrier are $0,2,4,6$ and 8 separately. So $b_{k, n} \in\{0,2,4,6,8\}$.

We study the relationship between ABSNR and number of bits assigned to each subcarrier. Without loss of generality, suppose $B E R=10^{-3},\left|h_{k, n}\right|^{2}=1$. We use (11) to get Figure 2 .

From Figure 2, we know that to assign 2 bits additionally will result in about $7 \mathrm{~dB}$ increment of transmit power. On the other hand, if channel gain increases five times, transmit power will reduce $7 \mathrm{~dB}$. As number of bits is selected from $\{0,2,4,6,8\}$, the median of the elements of $\{0,2,4,6,8\}$ is 4 .

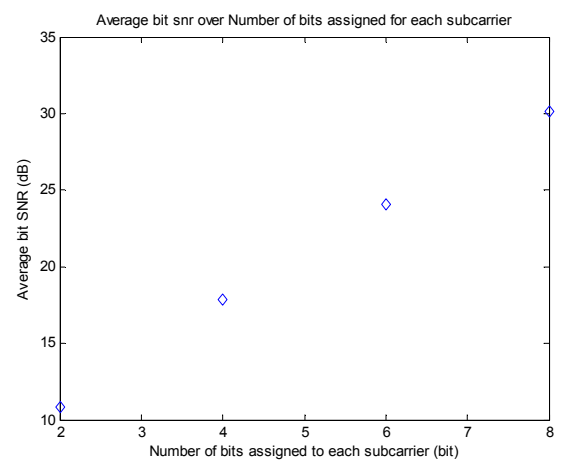

Fig. 2. ABSNR versus number of bits assigned for each subcarrier

Suppose the average channel gain is corresponding to the median. Then we assign the initial number of bits to subcarrier $n$ of user $k$ according to equation (19).

$$
b_{k, n}=\left\{\begin{array}{l}
6, \alpha_{\mathrm{k}}>5 \\
4,0.2 \leq \alpha_{\mathrm{k}} \leq 5 \\
2, \alpha_{\mathrm{k}}<0.2
\end{array}\right.
$$

The final number of bits assigned to subcarrier $n$ of user $k$ should be adjusted according to the requirement of minimum transmission rate of user $k$, but is limited to increase or reduce 2 bits with minimum transmit power. During the process of adjusting number of bits for $b_{k, n}, b_{k, n}$ is restricted to $\{0,2,4,6,8\}$. In the following we will do some simulations from several aspects to evaluate the performance of our algorithm.

First, to evaluate the performance, we compare our heuristic allocation algorithm (HAA) with fixed allocation algorithm (FAA), optimal allocation algorithm (OAA) and the algorithms proposed in [2] and [3] by average bit SNR with different number of users. Figure 3 gives the simulation results.



Fig. 3. The effect of number of users on average bit SNR

Figure 3 indicates that average bit SNR of our heuristic algorithm is close to that of optimal allocation algorithm with less than $2 \mathrm{~dB}$ losses, but much lower than that of fixed allocation algorithm with about $8 \mathrm{~dB}$ reduction of average bit SNR. Our heuristic algorithm achieves about $2 \mathrm{~dB}$ gain relative to the algorithms proposed in [2] and [3]. The number of users has little effect on average bit SNR.

Then we investigate the effect of minimum transmission rate on average bit SNR. Without loss of generality, suppose the required BER of all users are $10^{-3}$, number of bits per symbol assigned to each user is the same, number of users is 64, and then we get Figure 4. 
Figure 4 indicates that average bit SNR of our heuristic allocation algorithm is close to that of optimal allocation algorithm with about $2 \mathrm{~dB}$ losses, but much lower than that of fixed allocation algorithm with about $7 \mathrm{~dB}$ reduction of average bit SNR. Our heuristic allocation algorithm achieves about 1.8 $\mathrm{dB}$ relative to the algorithms proposed in [2] and [3]. The minimum transmission rate requirements of users have little effects on average bit SNR.

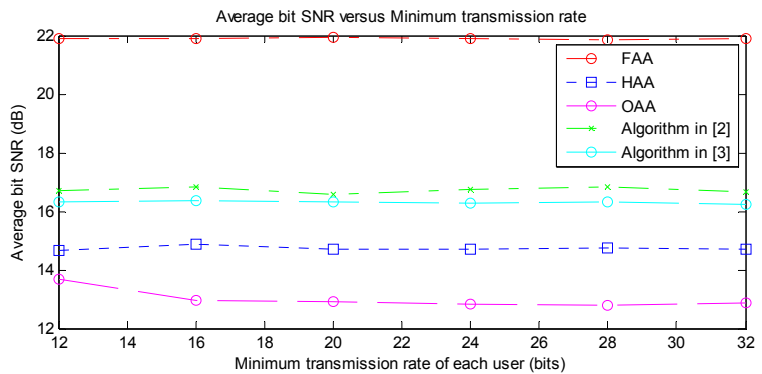

Fig. 4. The effect of minimum transmission rate on average bit SNR

Last, we study the relationship between BER and average bit SNR. We suppose the requirements of BER and minimum transmission rate are the same for all users, number of users is 64 , and other simulation parameters are given by Table I. Figure 5 gives the simulation results.

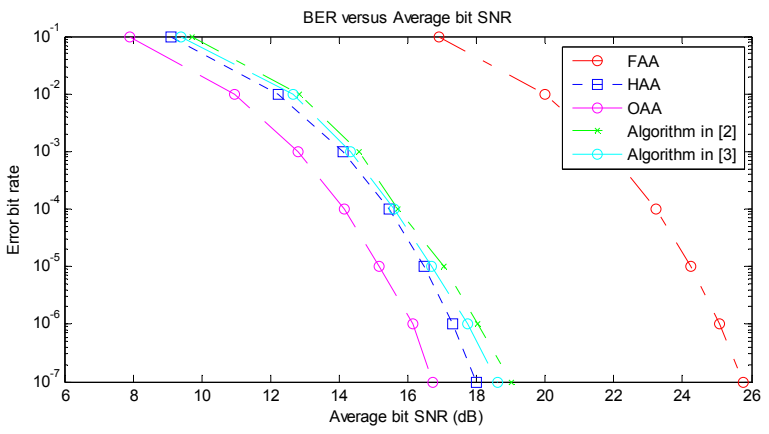

Fig. 5. BER over Average bit SNR for several algorithms

Figure 5 indicates that given the same BER, our heuristic allocation algorithm acquires about $8 \mathrm{~dB}$ gain relative to fixed allocation algorithm, about $0.5 \mathrm{~dB}$ gain relative to the algorithms proposed in [2] and [3], and less than $2 \mathrm{~dB}$ losses relative to Optimal allocation algorithm.

\section{Analysis of Algorithm's Complexity}

Though fixed allocation algorithm (FAA) is simplest, its performance is worst. Now we compare the complexity of our heuristic allocation algorithm (HAA) with that of optimal allocation algorithm (OAA).

Comparing with OAA algorithm, HAA algorithm estimates number of subcarriers assigned to each user firstly according to the requirement of minimum transmission rate for all users, and need not search all the channel gains exhaustively. Furthermore, when loading bits for each subcarrier, HAA algorithm pre-assigns number of bits for each subcarrier according to the ratio of its channel gain to the average channel gain of all users; this also benefits reduction of search number.

\section{CONCLUSION}

In this paper we propose a heuristic algorithm of subcarrier, bit and power assignment, and compare average bit SNR of our heuristic allocation algorithm with that of fixed allocation algorithm, optimal allocation algorithm and the algorithms proposed in [2] and [3]. Simulation results indicate that our heuristic algorithm achieves asymptotic performance of optimal allocation algorithm with much lower complexity. Given the same BER, our heuristic algorithm achieves about $8 \mathrm{~dB}$ gain relative to fixed allocation algorithm, about $0.5 \mathrm{~dB}$ gain relative to the algorithms proposed in [2] and [3] and about $1.5 \mathrm{~dB}$ loss relative to optimal allocation algorithm. Though we just consider two classes of services in the simulation, it is easy to extend to more classes of services without increasing complexity.

\section{References}

[1] Feng She, Wen Chen, Hanwen Luo, Tingshan Huang, and Xinbing Wang, "Joint Power Allocation and Scheduling of Multi-antenna OFDM System in Broadcast Channel," ICC 2009

[2] KHALID EL BAAMRANI, ABDELLAH AIT OUAHMAN, VICTOR P. GIL JIM 'ENEZ, ANA GARCIA ARMADA and SAID ALLAKI, "Subcarrier and Power Allocation for the Downlink of Multiuser OFDM Transmission," Wireless Personal Communications (2006) 39: 457-465

[3] HANDONG GONG, WU YE, SUILI FENG and HUI SONG, “A Subcarrier Allocation Algorithm for Efficiently Reducing Power in Multiuser OFDM Systems," Wireless Personal Communications (2007) 40: 233-243

[4] Cheong Yui Wong, Roger S. Cheng, Khaled Ben Letaief, and Ross D. Murch, "Multiuser OFDM with Adaptive Subcarrier, Bit, and Power Allocation," IEEE JOURNAL ON SELECTED AREAS IN COMMUNICATIONS, VOL. 17, NO. 10, OCTOBER 1999, $1747-1758$

[5] Guanding $\mathrm{Yu}$ - Zhaoyang Zhang · Peiliang Qiu, "Adaptive subcarrier and bit allocation in OFDMA systems supporting heterogeneous services," Wireless Personal Communications (2007) 43:1057-1070

[6] Chongxian Zhong, Chunguo Li, Rui Zhao, Luxi Yang, Xiqi Gao, "Dynamic Resource Allocation for Downlink Multi-user MIMO-OFDMA/SDMA Systems," ICC 2009

[7] Winston W. L. Ho, Ying-Chang Liang, "Optimal Resource Allocation for Multiuser MIMO-OFDM Systems with User Rate Constraints," IEEE TRANSACTIONS ON VEHICULAR TECHNOLOGY, VOL. 58, NO. 3, MARCH 2009, 1190-1203

[8] Ying Jun Zhang, Khaled Ben Letaief, “An Efficient Resource-Allocation Scheme for Spatial Multiuser Access in MIMO/OFDM Systems," IEEE Transactions on Communications, VOL. 53, NO. 1, JANUARY 2005, 107-116

[9] Young-il Shin, Tae-Sung Kang, Hyung-Myung Kim, "An Efficient resource Allocation for Multiuser MIMI-OFDM Systems with Zero-Forcing Beamformer," PIMRC 2007

[10] Ya-Han Pan, Khaled Ben Letaief, and Zhigang Cao, "Dynamic Spatial Subchannel Allocation with Adaptive Beamforming for MIMO/OFDM Systems," IEEE TRANSACTIONS ON WIRELESS COMMUNICATIONS, VOL. 3, NO. 6, NOVEMBER 2004, 2097-2107

[11] 9. J.G. Proakis, Digital Communications, 4th ed. Beijing: Publishing House of Electronics Industry, 2001. 